\title{
RIVALIDAD Y SORORIDAD FEMENINA EN EL SECTOR HOTELERO DE TOLUCA, MÉXICO ${ }^{1 *}$
}

\author{
Rebeca Mejía-Vázquez* \\ Universidad Autónoma del Estado de México \\ https://orcid.org/0000-0002-4541-7286 \\ Rocío del Carmen Serrano-Barquín* \\ Universidad Autónoma del Estado de México \\ http://orcid.org/0000-0002-5695-751X \\ María José Pastor-Alfonso** \\ Universidad de Alicante \\ https://orcid.org/0000-0001-8820-8073
}

\section{RESUMEN}

El objetivo de la investigación es analizar las relaciones intragenéricas, principalmente entre el género femenino, en trece hoteles de Toluca, México. Es una investigación de carácter cualitativo, además se realiza a partir de la perspectiva de género y el vínculo entre las dimensiones cultura, cuerpo y poder. En el trabajo se destaca el departamento de ama de llaves, donde la performance que se vive se vincula con las características socioculturalmente establecidas para lo femenino. Además, se observa que la relación entre mujeres puede ser conflictiva y difícil; sin embargo, en ocasiones puede llegarse a relaciones de sororidad.

Palabras clave: Turismo; género; sororidad; hotelería.

Fecha de recepción: 31 de mayo de 2019.

Fecha de aceptación: 15 de julio de 2020.

1 Los hallazgos son parte de una tesis de Maestría en Estudios Turísticos de la Universidad Autónoma del Estado de México.

* Centro de Investigación y Estudios Turísticos. Facultad de Turismo y Gastronomía. Universidad Autónoma del Estado de México. Cerro de Coatepec s/n. Ciudad Universitaria. C.P. 50110. TOLUCA. Estado de México (México).E-mail: mejiarbk@gmail.com,rocioserba14@gmail.com

** Facultad de Filosofía y Letras, Departamento de Humanidades Contemporáneas. Universidad de Alicante. Carretera San Vicente del Raspeig s/n. 03690 San Vicente del Raspeig. ALICANTE (España). E-mail: josefa.pastor@ua.es 


\title{
Rivalry and female sorority in hosting companies of Toluca, México²
}

\begin{abstract}
The aim of this research is to analyze intrageneric relations, mainly the female gender relations, in thirteen hotels in Toluca, Mexico. The research is carried out from the gender perspective and the link between the dimensions of culture, body and power. Qualitative method was employed. In the research, the housekeeper area stands out, where the performance that they live is related to the socio cultural characteristics for the female gender. In addition, the results of this research indicate how the relationship between women is, which can become conflictive and difficult; however, sometimes sorority can be reached.
\end{abstract}

Keywords: Tourism, gender, sisterhood, hosting companies.

\section{INTRODUCCIÓN}

Las relaciones de género (referidas a relaciones de poder) tanto inter como intragenéricas desempeñan un rol importante en la sociedad, debido a que no solo están presentes en la evolución y transformación de las normas y valores, sino además están inmersas en la vida cotidiana de las personas. En este sentido, el tema de relaciones de género se ha estudiado en diferentes contextos. Por ejemplo: en la universidad (Rojas, Méndez y Montero, 2013), sector salud (Martínez, 2014), familia (Ribeiro, 2004, Navarro, 2017), migración (Rendón, 1997), dinámicas del cambio cultural en contextos indígenas (Rodríguez, 2011) y en el turismo (Fuller, 2010; Mendoza y González, 2013; Díaz-Carrión, 2014; Díaz, Lozano y Montiel, 2018).

$\mathrm{El}$ análisis que se ha realizado en estos contextos se ha hecho desde las relaciones intergenéricas (mujer-hombre). El objeto de estudio se vincula con la situación y las diversas condiciones que viven las mujeres; es decir, qué roles se les han asignado, la opresión contra ellas (Sánchez, 1995; Blanco, 2006; Ramalio, 2011, Martínez, 2014), así como las condiciones de desigualdad (Costa, Carvalho y Breda, 2011) y obstáculos socioculturales para ocupar puestos de mayor jerarquía en el ámbito laboral (Boone, Veller, Nikolaeva, Keith, Kefgen y Houran, 2013; Díaz, Lozano y Montiel, 2018; Carvalho, Costa, Lykke y Torres, 2019). Además, la mujer se encuentra en desventaja, por ejemplo, en las condiciones de trabajo de las organizaciones del sector turístico (Santero-Sanchez, Segovia-Pérez, Castro-Núñez, Figueroa-Domecq y Talón-Ballestero, 2015; Mooney, Ryan y Harris, 2017). Por otra parte, los estudios sobre las relaciones intragenéricas en el turismo ha sido menor, en especial cuando se refiere a relaciones entre el género femenino (mujer-mujer). Por esta razón, es esencial adoptar un enfoque que considere la manera en que las mujeres experimentan y viven su participación en el turismo (Díaz, 2010) desde este tipo de relaciones.

\footnotetext{
2 The findings are part of a Master's thesis in Tourist Research of Universidad Autónoma del Estado de México.
} 
En cuanto a la incorporación de la mujer en el sector, el turismo genera millones de empleos para ellas en todo el mundo (Sarosi, 2017). Esto se refleja en que el personal en turismo está integrado en su mayoría por mujeres (UNWTO, 06 de marzo de 2019). Por ejemplo, en América Latina, la plantilla laboral femenina en el sector de Hoteles y Restaurantes representó 58.5\% y, en el Caribe 53.4\% (OMT y ONU Mujeres, 2013). Por otra parte, la participación de mujeres en la actividad económica de servicios en México fue de 53,7\% (INEGI, 2018), mientras que en el Estado de México representó 54.5\% (Gobierno del Estado de México, 2018).

En este estudio se consideraron los hoteles debido a que son empresas turísticas donde la división de género es evidente (Sarosi, 2017; Mejía et al., 2017; Escudero et al., 2017). Además, son espacios que permiten estudiar la performance, entendida como el acto, la representación de los cuerpos y del género (Butler, 1998) entre el colectivo femenino. Es importante mencionar que el desarrollo de la actividad y la incorporación de la mujer en el empleo turístico han generado cambios, han acelerado el proceso de diversificación de la economía familiar y de la composición social de la población (Fuller, 2010, VizcaínoSuárez et al., 2017), y han influido en las relaciones entre los géneros.

En el caso de las mujeres, su vida está marcada por ideas patriarcales: la competencia, la exclusión, la discriminación y todas las formas de opresión (Lagarde, 2012). Esto no solo se vive en México, sino que se manifiesta en naciones con contextos similares donde el sistema patriarcal es dominante. Sin embargo, este tipo de situaciones no solo se refleja entre diferentes géneros (masculino-femenino), sino también en la interacción entre ellas (mujer-mujer). Por ejemplo, su relación social resalta en aspectos de competencia, rivalidad, disputa. No obstante, también se identifican situaciones de sororidad, hermandad y lucha del bien común.

En esta investigación se toman en cuenta las relaciones intragenéricas (entre mujeres), la relación histórica entre ellas con base en su enemistad, donde lo común es anulado y solo queda aquello que las separa, que mantiene a las mujeres en solitaria disputa (Lagarde, 2012). ¿Incapaces y sin posibilidad de alianza y pacto o con opción de incorporar la sororidad en los espacios del sector turístico donde desempeñan un rol? La sororidad se considera una opción de alianza, la cual está basada en una relación de amistad, de hermandad entre mujeres, donde se aprende y enseña. Es decir, en las amigas mujeres se encuentra una persona a quien se acompaña y con quien se construye (Pérez, 2009). Además, en este tipo de interacciones de sororidad se modifican las relaciones entre ellas para lograr relaciones sociales favorables para cada una de las que integra el colectivo femenino.

En este sentido, sororidad es un tema reciente, la palabra entró en el diccionario en línea de la RAE en 2018. También destaca la propuesta de Marcela Lagarde (2006) quien incorpora este concepto en español. En general, este término es cada vez más relevante (BBC Mundo, 2018). Ejemplo de ello es lo que sucede en redes sociales con los hashtags \#yotecreo, \#metoo (Quintana, 17 de octubre de 2018) y \#sororitips. Se busca dar voz a las mujeres y es una forma de identificarse y ayudarse entre ellas mismas. Un caso en el turismo es el que sucede con las camaristas, camareras de piso, las que limpian los hoteles (las Kellys), quienes han hecho que su voz se escuche sobre su condición y situación de trabajo a través de la creación de asociaciones en diferentes lugares de España (Cañada, 2015; Durán, 22 de enero de 2016) para dejar de ser invisibles (Las Kellys, 1 de diciembre de 2018). 
Con base en lo anterior, la investigación que da soporte a este trabajo tiene lugar en las empresas de hospedaje de Toluca, México, con el objetivo de analizar las relaciones de género, principalmente intragenéricas (entre mujeres), a partir del vínculo entre las dimensiones de la perspectiva de género: cultura, poder y cuerpo. El trabajo se construyó desde los siguientes apartados: el primero está destinado a la introducción; en el segundo se exponen los fundamentos teóricos que dan pie a la discusión; posteriormente se presenta la metodología; seguido de los resultados y, el último apartado está destinado a las conclusiones.

\section{RELACIONES DE GÉNERO Y TURISMO}

Los estudios sobre género en el turismo son relativamente recientes, es en los noventas cuando este tipo de investigaciones tiene apertura de manera internacional; por ejemplo, con la publicación "Gender in tourism" de Swain (1995). Aunado a lo anterior, la investigación de género en turismo parece un territorio incómodo, un terreno marginalizado para las mujeres y un espacio extraño para los hombres (Pritchard y Morgan, 2017). Existen estudios donde la implicación de esta perspectiva se asocia con el análisis en el ámbito laboral de las relaciones desiguales de poder entre el género femenino y el masculino (Costa et al., 2011; Boone et al., 2013; Munar et al., 2015; Figueroa-Domecq, Pritchard, Segovia-Pérez, Morgan y Villacé-Molinero, 2015; Walters, 2018).

No obstante, el número de investigaciones sobre las relaciones intragenéricas en el turismo ha sido menor, aunque algunas de ellas centran su análisis y explicación de la relación entre el colectivo femenino a través de su empoderamiento (Ferguson, 2010; Mendoza y Chapulín, 2015; Vizcaíno-Suárez et al., 2017), todavía existe un área de oportunidad para analizar las relaciones sociales entre mujeres.

En este contexto, se considera que la perspectiva de género ayuda a entender la posición de las mujeres en la industria hospitalaria (Segovia-Pérez, Figueroa-Domecq, Fuentes-Moraleda y Miñoz-Mazón, 2019), así como las relaciones de género, las cuales pueden ser entendidas como relaciones de poder jerarquizadas entre mujeres y varones (Vizcaíno-Suárez et al. 2017) o entre sujetos del mismo género (por ejemplo, entre el colectivo femenino). En ambas interacciones intervienen tres aspectos: la cultura, el cuerpo y el poder (Tepichin, Tinat y Gutiérrez, 2010). En este tipo de interacciones, se observa el papel del turismo como filtro del poder y de la cultura y, de cómo estas dos dimensiones atraviesan el cuerpo.

Por esta razón, las relaciones de género desempeñan un rol importante al estar presentes en la evolución y transformación de las normas y valores de la sociedad y de alguna manera en todas las esferas de la convivencia. Es a partir de la perspectiva de género que se pueden observar las complejas y diversas relaciones sociales que se dan al interior de uno de los géneros. En la Figura 1, se aprecia la propuesta del modelo de análisis y la categoría de relaciones intragenéricas (entre el género femenino) que se analizó en esta investigación. 


\section{Figura 1 \\ MODELO DE ANÁLISIS}

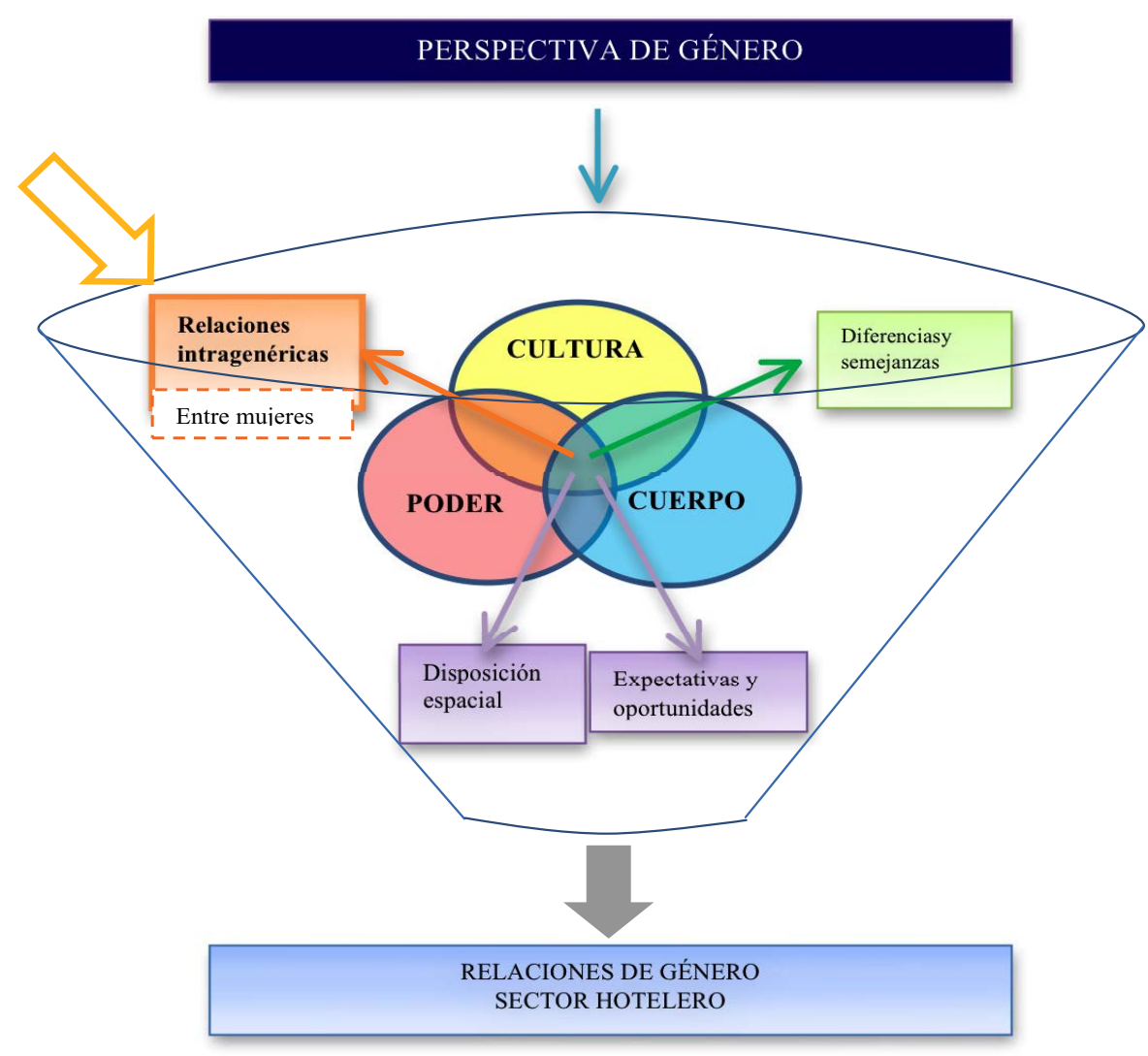

Fuente: Elaboración propia con base en Tepichin, Tinat y Gutiérrez (2010), Lamas (2017), Amigot y Pujal (2009).

Para entender el modelo de análisis y su vínculo con el turismo es importante explicar las tres dimensiones que integran el modelo. Por una parte, el poder es incomprensible si antes no se entiende al sujeto y su contexto, esto considerando que cuando el sujeto mantiene relaciones con otras personas y propiamente con la sociedad, se presentarán y desarrollarán relaciones de poder, que permiten comprender los contextos sociales (Foucault, 1991). Igualmente, puede ser un productor y regulador de las prácticas cotidianas y de la vida social (Amigot y Pujal, 2009). Asimismo, puede estar presente en la asignación de las características de lo masculino y lo femenino, así como de las funciones y responsabilidades de cada uno. Estas asignaciones se derivan del patriarcado, como voluntad de poder. El patriarcado se vincula a la cultura occidental donde se sobrevaloran los aspectos masculinos, y el ideal masculino se erige como una manera superior de comportamiento vinculado con estilos particulares de control y poder social (Lara, 1993). 
Estos procesos de construcción sociocultural acompañan a los individuos desde su nacimiento y están establecidos de acuerdo a patrones de masculinidad y feminidad, son productos culturales que se construyen socialmente (Beauvoir, [1949], (2017) ). Se pueden identificar, en algunos casos, a partir de la vestimenta, poses, comportamiento (Núñez, 1999), modalidades de crianza y juegos propuestos en la infancia (Indina, 2016), así como la interacción con otras personas del mismo o diferente género. Cada cultura concede significados distintos sobre la diferencia de género, donde la construcción de la sexuación de los cuerpos ${ }^{3}$ estructura los usos y costumbres particulares, y contrasta los mandatos culturales (Lamas, 2017); es decir, en cada cultura se otorga cierto significado a los cuerpos de las mujeres y de los hombres. Estos significados se reflejan en la asignación de puestos en el ámbito laboral del sector turístico (Costa, Carvalho y Breda, 2011).

Aunado a lo anterior, en dicha asignación, también se toman en cuenta los rasgos establecidos para el género masculino (enfocados a lo práctico, de orientación a la acción, a la protección, a ser proveedor, así como al machismo y a la agresividad); mientras que los rasgos femeninos se asocian a la preocupación por otros, de relación, maternidad, al síndrome de mujer sufrida o sumisión — debilidad - (Lara, 1993), y con actividades del hogar (Fernández y Martínez, 2010; Cacique, 2008).

La vida de las mujeres está definida por esta voluntad de poder patriarcal y está marcada por la competencia, la exclusión, la discriminación y todas las formas de opresión. Al vivir, ellas las reproducen, son sus portadoras. Por ejemplo, en una encuesta realizada por Valen (2010) sobre la relación de las mujeres, la mayoría respondió que la influencia de la madre es el indicador clave sobre si una mujer llega a ser agresiva, manipuladora o exclusiva con sus compañeros.

Continuando con esta línea, Valen (2 de diciembre de 2007) señala que el miedo sobre la competencia y agresión encubiertas de las mujeres se valida con frecuencia en el chisme, las comparaciones, así como en las críticas fulminantes de la carrera. Aunado a eso, a menudo las mujeres no tienen una idea de dónde se encuentran - socialmente, como madres, colegas - porque al mismo tiempo son aliadas y enemigas.

Hablar de una diferencia histórica anclada en un cuerpo vivido (Beauvoir, [1949], (2017) ) es suma específica de la historia de las mujeres, donde los cuerpos trabajan, crean, transmiten y recrean la cultura, los modos de vida y las relaciones de poder; no se trata de cuerpos biológicos sino de cuerpos construidos por las relaciones entre biología (sexo), sociedad, cultura (Lagarde, 2012) y poder. El cuerpo entendido como el espacio o un territorio propio que el sujeto, tanto hombre como mujer, pueden controlar y dominar. Es una construcción de género y un soporte de las clasificaciones de lo femenino y de lo masculino (Tepichin, Tinat y Gutiérrez, 2010). Es decir, el cuerpo es la entidad que refleja la representación (performance) sociocultural con base en el género (hombre-masculino / mujer-femenino).

Este tipo de relaciones, aspectos, performance y asignaciones se asocian a un liderazgo con la etiqueta de "valores masculinos", los cuales son adoptados por algunas mujeres en puestos gerenciales o de mayor jerarquía en la hotelería (Mooney, Ryan y Harris, 2017;

3 A partir no solo de lo biológico sino también de lo psíquico y social se construye lo "propio" para los varones y lo "propio" para las mujeres. 
Segovia-Pérez et al., 2019). Aunque se opta y sugiere trabajar con estilos de liderazgo efectivamente neutros al género (Díaz, Lozano y Montiel, 2018), las mujeres, ante su necesidad de poder, intentan identificarse con el hombre (Lagarde, 2012).

No obstante, se propone la no existencia del hombre universal, sino la de hombres y mujeres culturalmente diferentes (Harding, 1988), no sólo entre los géneros, sino también entre el mismo género, en este caso, entre mujeres. En este sentido, Lagarde (2012) menciona que la diferencia para las mujeres es doble. Por una parte, se es diferente en relación con los hombres como paradigma patriarcal y estereotipo de lo humano y, por otro lado, se encuentra la diferencia de cada una con relación a las otras; es decir, de lo que las mantiene en disputa. La disputa entendida como algo histórico, lo común es anulado y sólo queda entre las mujeres aquello que las separa - clases, grupos de edad, relación con los hombres, con los otros y con el poder, color, belleza, rango, prestigio-, es decir, lo que está en la base de su enemistad histórica (Lagarde, 2012).

Por otra parte, se encuentra el feminismo como otra voluntad de poder, el cual es concebido como el movimiento social que cuestiona valores, creencias y normas en la sociedad que destinan a las mujeres roles subordinados respecto a los varones (Montero, 2006; Jaiven, 2016). Un elemento que incorpora esta voluntad es la experiencia de sororidad (sisterhood en inglés y sororité en francés); es decir, de compañerismo, amistad y/o parentesco entre mujeres, que se convierte en nuevas pautas de relación entre conocidas y desconocidas (Riba, 2016). También, se suscribe en todo escenario donde las mujeres tengan convivencia o interrelaciones (Pérez-Sánchez y Rábago-De Ávila, 2018).

En la práctica, las relaciones de sororidad entre mujeres se identifican en asociaciones como Las Kellys y Tourism Sisterhood Nepal Association - TSNA - que comparten objetivos vinculados con reducir la brecha de género y mejorar las condiciones y oportunidades de trabajo de las mujeres que laboran en el sector turístico. Después de la publicación del artículo sobre las mujeres que limpian los hoteles (Cañada, 30 de julio de 2014), las camaristas de España comenzaron a compartir sus historias (algunos de los temas fueron acerca de la explotación, falta de valor atribuido a su trabajo, así como de las condiciones precarias en las cuales trabajan). "Las Kellys Federadas" es un ejemplo de trabajo con elementos de sororidad porque ha tratado de cambiar la percepción acerca del puesto que desempeñan las camaristas en los hoteles, pero no solo ha sido eso, también ha contribuido en la relación de trabajo entre el colectivo femenino, donde a partir de situaciones comunes, se genera un ambiente de sororidad para obtener mejores condiciones de trabajo, así como la valorización de sus actividades laborales, el fortalecimiento de su autoestima y con ello su empoderamiento. De igual forma, ayudan a develar los desafíos para la elaboración de políticas con perspectiva de género (inclusivas y sostenibles) en el sector turístico (UNWTO, 2019).

Es por ello que el espacio donde llevan a cabo una performance y donde se presentan las interacciones sociales entre ellas puede ser un lugar donde también se visualice el tema de sororidad que "vincula, mueve y sostiene a las mujeres" (Riba, 2016: 230). También, da pauta para que en la sororidad se encuentre la posibilidad de desarticular, además de la enemistad histórica mujer-mujer, la opresión patriarcal entre los géneros hombre-mujer (Lagarde, 2012), situación que se vive en la industria de la hospitalidad del sector turístico. 


\section{METODOLOGÍA}

Para este artículo se optó por un análisis de carácter cualitativo. El trabajo de campo se realizó de junio a noviembre de 2018. En primera instancia se limitó el estudio a hoteles entre una y cinco estrellas de Toluca, México. Posteriormente, se tomó en cuenta que los dueños o gerentes de cada hotel autorizaran su participación en la investigación. Entonces, con base en el Directorio de Hospedaje del Estado de México (Sectur Edo. Méx., 2018), en Toluca están registrados 26 alojamientos que cumplen con el primer criterio de la categoría. Sin embargo, de acuerdo con el segundo elemento (autorización), solo trece establecimientos participaron en el estudio, debido a que el resto de las empresas por motivos de sus políticas de privacidad no podían brindar información acerca de sus empleados (salario, tipo de puestos), ni tampoco permitían realizar entrevistas al personal de trabajo.

A continuación, se aplicó un cuestionario a cada Gerente General o Gerente de Recursos Humanos de los 13 hoteles que participaron en este trabajo para conocer la distribución del personal (Martínez, 2004; Martín, 2009; Buquet, Cooper, Mingo y Moreno, 2013).

En la investigación se utilizaron técnicas del método etnográfico (entrevistas semiestructuradas, conversaciones informales, observación participante y no participante) debido a que es un método de campo que se realiza en los entornos de las personas a estudiar y con quien se tiene contacto cara a cara (Angrosino, 2012). En este sentido, no se buscó representatividad estadística, sino información diversa y rica sobre las relaciones sociales entre mujeres. La muestra estuvo compuesta por 26 entrevistas semiestructuradas (18 mujeres y 8 hombres). Con dicho número de entrevistas (tanto para mujeres como para varones) se alcanzó el nivel de saturación de la información (Vallés, 2009).

Primero se entrevistó a las mujeres y hombres que ocupaban puestos como gerente, jefe (a) o encargado (a) de área. Posteriormente, ellos y ellas indicaban a quién entrevistar (qué área de trabajo) porque tomaban en cuenta las actividades y horarios de trabajo de las personas. Por ejemplo, algunas entrevistas a camaristas se realizaron mientras hacían sus actividades laborales (limpieza de habitaciones). Es importante mencionar que se solicitó permiso para entrevistar al personal de todas las áreas del hotel. En los resultados, no se usa el nombre de las personas por motivos de privacidad, en su lugar se pone el puesto que desempeñan en la empresa de hospedaje, el mes y año de la entrevista. Además de las entrevistas, se recurrió a la observación participante (prácticas profesionales en dos hoteles, del 13 de agosto al 19 de octubre de 2018), a la observación no participante y a las conversaciones informales.

Para la recogida de información de las técnicas anteriores se usó grabadora de voz digital, libreta de notas y cédulas de observación. Después, la información obtenida se organizó y analizó a través de su codificación e interpretación (Gibbs, 2007). En este artículo, el nivel de codificación se centró en la categoría "relaciones intragenéricas"; para ello, se transcribieron las entrevistas, posteriormente, se identificó cómo es la relación entre personas del mismo género y qué área de trabajo predominaba a partir de las respuestas obtenidas. Entonces, los códigos para su interpretación se agruparon: 1) entre quiénes (hombres-hombres, mujeres-mujeres), 2) tipo de relaciones (disputa, sororidad) y, 3) área de trabajo. Con base en lo anterior, los resultados se concentraron en la relación entre mujeres, principalmente de quienes trabajan en el área de ama de llaves. De acuerdo 
con las entrevistas realizadas, se consideró este puesto porque durante las entrevistas se identificó como un área de "disputas y conflictos" entre mujeres debido a que está integrado, en su mayoría, por mujeres.

\section{RESULTADOS}

De acuerdo con la primera etapa del trabajo de campo, y con base en los resultados del cuestionario sobre la distribución del personal aplicado en las 13 empresas, se obtuvo que $50.7 \%$ del personal que labora son mujeres y $49.3 \%$ son hombres; situación similar a lo que sucede en la región de América Latina y el Caribe (UNWTO, 2018), en el sector servicios en México (INEGI, 2018), y en el Estado de México (Gobierno del Estado de México, 2018), donde la participación femenina es mayor. En este tenor, la distribución del personal femenino en las diferentes áreas que integran a las empresas de hospedaje de Toluca, México que participaron en la investigación, se visualiza de la siguiente manera (véase figura 2).

\section{Figura 2 \\ DISTRIBUCIÓN DEL PERSONAL FEMENINO EN LAS EMPRESAS OBJETO DE ESTUDIO (TOLUCA, MÉXICO)}

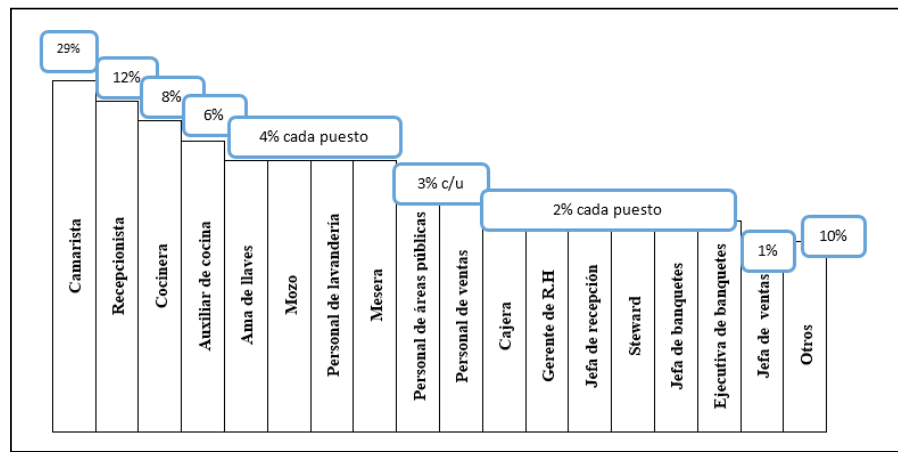

Fuente: Elaboración propia con base en resultados (2018).

El área de trabajo "otros" está compuesto por espacios donde la representación de la mujer es de $1 \%$ en cada uno. Por ejemplo: gerente general, contadora, gerente servicio a huésped, personal de cuentas por cobrar, entre otros. Como se observa en la Figura2, los puestos con mayor participación de mujeres son camarista, recepcionista, personal de cocina y ayudante de cocina; en este sentido, las actividades que desempeñan están vinculadas con actividades del hogar (Fernández y Martínez, 2010; Cacique, 2008), principalmente con la limpieza, preparación de alimentos, atención y cuidado de las personas. Como sucede, por ejemplo, en el sector turístico de México (Bañuelos, Huesca y Horbath, 2017; Mejía et al., 2017), en el turismo alternativo (Díaz-Carrión, 2013) y en el turismo rural de la Costa Coruñesa (Sparrer, 2003).

Aunado a lo anterior, durante las entrevistas, se les preguntaba sobre cómo era la relación social entre varones y mujeres; cuando ejemplificaban acerca de la interacción entre 
mujeres mencionaban el área de camaristas y describían su convivencia como conflictiva. Por ejemplo, una Gerente de Recursos Humanos, quien había trabajado seis años en el sector automotriz (la plantilla del personal en esa empresa estaba compuesta por varones) mencionó que, para ella es un desafío trabajar ahora con mujeres, debido a las situaciones que en ocasiones se presentan en el área de ama de llaves. Entre camaristas se generan conflictos, además, al ser un puesto integrado por más de diez mujeres, la dinámica de trabajo y la relación se vuelven diferentes.

Las camaristas son súper difíciles, «que ya agarró el trapo de mi carrito, que ya tomó un agua y me dijo que la iba a reponer y se le olvidó, por equis razón ya no me la trajo», "es que yo escuché que mi compañera dijo esto», cuando en realidad ni siquiera sucede eso (Gerente de Recursos Humanos, julio 2018).

El área de ama de llaves, al ser un equipo de trabajo donde el personal que lo representa es en su mayoría mujeres se llegan a dar más casos asociados con conflictos. Así, "cuando no hay un equilibrio y hay puras mujeres, yo creo que sí se hacen daño y los daños a veces son absurdos, pero dejan marcada a la gente" (Gerente operativo, julio 2018). Por una parte, es como si se viviera en disputa, se suprimiera lo común (se puede vincular a los rasgos femeninos, entre ellos, la preocupación por otros) y, quedara solo entre ellas aquello que las separa.

Pues yo creo que es más complicado llevarnos entre mujeres porque normalmente es como «no, es mi idea, yo quiero que mi idea se haga asi y asi»», "pero también tienes que escuchar la mía» (Ayudante general, julio 2018).

Aunado a lo anterior, durante las prácticas profesionales en un hotel, una camarista a quien se le ayudó en sus actividades laborales, compartió que su trabajo estaba bien (actividades, horarios), pero empezó a bajar la voz, cerró la puerta de la habitación que estábamos limpiando, y después comentó que lo único que no le gustaba era que sus compañeras le dejaban más habitaciones y, eso lo asociaba a que ella era nueva, tenía menos de tres meses en el puesto, “a las novatas nos pasa eso” (trabajo de campo, octubre 2018). ¿Por qué surgen este tipo de acciones por parte de las mujeres? La respuesta podría referirse al ritmo de trabajo que tienen en este puesto, pero de ser así, la asignación de habitaciones sería igual, independientemente de si es de nuevo ingreso o ya tiene tiempo trabajando ahí. En este caso, la camarista que compartió su sentir sobre su disgusto, lo asociaba a una forma de poder, porque cuando llegara una camarista nueva, ella ya no sería la novata. Entonces, le tocarían el mismo número de habitaciones que sus demás compañeras y la de nuevo ingreso ocuparía "el rol que ahora ella tiene".

Situación que no se presentó cuando contrataron a un varón en el mismo puesto, al contrario, le asignaron menos habitaciones para su limpieza. En una plática con las camaristas durante la hora de comida, comentaron que estaban agradecidas porque había un varón en su departamento y él podía ayudarlas con actividades que requerían fuerza; por ejemplo, llevar la lencería (toallas, sábanas) tanto a la lavandería como a las habitaciones. Además, como él no estaba acostumbrado a ese tipo de actividades (limpiar, tender camas, lavar 
baños), para ellas era de gran ayuda que él limpiara, aunque fueran solo siete habitaciones (ellas en promedio limpiaban entre 11 y 13 habitaciones diarias).

Por otra parte, una mujer que ahora se desempeña como ama de llaves compartió que para ella no había sido fácil aceptar el puesto de ama de llaves porque sus compañeras de área no estaban de acuerdo con la decisión de su ascenso. Como parte del contexto, las camaristas expresaron que su jefa era una persona fuerte y estricta.

Mira, creo que todo humano somos humillados cuando vemos crecer a una persona tratamos de que no lo haga, que se vaya al suelo y en cuestión de eso, es hablar mal de la persona o decir "por qué le dieron ese puesto a ella, no se lo merecía» o así, cosas negativas (Ama de llaves, julio 2018).

En esta situación se observa lo que está en la base de su enemistad histórica cuando una de ellas ocupa puestos de mayor jerarquía. En este caso, la disputa se vincula con el rango que se tiene en una organización y el miedo sobre la competencia se valida en las comparaciones por parte de sus compañeras hacia ella, así como en la crítica y expresiones acerca de no merecer ese puesto. ¿Qué pasa cuando un varón ocupa un puesto de mayor jerarquía? Por parte de las mujeres se genera una aceptación porque está vinculado con "valores masculinos", esta relación hombre-jefe como algo normal. Por ejemplo, en las entrevistas expresaban que la mayoría de puestos de jefatura son ocupados por hombres y en el caso de algunas mujeres "es pesado (difícil) y dejas de lado mucho a la familia" cuando eres jefa o gerente general (Gerente de Recursos Humanos, junio 2018).

Con referencia a lo anterior, un gerente general comentó que las relaciones entre mujeres y entre hombres se puede vivir diferente, quizá porque en la infancia, ellos se involucraban en juegos de competencia, juegos de equipo con otros niños (fútbol, canicas). Mientras que las niñas estaban en casa y jugaban a las muñecas. Estos eventos se asocian a los procesos de construcción sociocultural que acompañan a los individuos desde su nacimiento y se pueden identificar en las relaciones con otras personas. No obstante, en este contexto, se consideraron solamente las relaciones entre el colectivo femenino, por lo cual, para futuras investigaciones, se exhorta a continuar con esta línea de estudio para examinar y revisar cómo sería el rol y la relación entre varones.

En otra empresa de hospedaje, la encargada de recepción comentó que su jefa anterior (gerente de operaciones) al principio era muy cerrada y estricta, características vinculadas al género masculino:

Después empezó a ceder en ciertas cosas, pero yo siento que era como de esas personas que tienen como una obsesión por el poder, que realmente a pesar de que estamos: ama de llaves, el jefe de mantenimiento, yo como jefa de recepción, o jefa de cocina, si tú le decías algo era como de "ah sí, lo voy a considerar», pero realmente nunca nos dejaba hacer nada, o sea era de «todo yo, yo, yo» (Jefa de recepción, julio 2018)

Sobre la base de las consideraciones anteriores, se observa la doble diferencia y disputa, por una parte, en relación con los hombres (adoptar un liderazgo con la etiqueta de 
"valores masculinos") y por otra con las mujeres (disputa vinculada con el rango); además, incide en la performance de lo cultural y en las relaciones de poder. En este sentido, un varón comentó que es complicado cuando una mujer "ejerce poder" refiriéndose a ocupar un puesto de jefatura, porque él había percibido que su jefa en algunas ocasiones llegaba a tratarlo mal; es decir, le gritaba o lo presionaba para hacer sus actividades, y esa situación es más notoria cuando está presente otra mujer. En otras palabras, cuando había una mujer mientras su jefa hablaba con él, su jefa se alteraba más y era más estricta, como si necesitara demostrar que en ese momento la diferencia hombre-mujer no está presente y ella no es vulnerable, sino al contrario, puede ejercer poder a partir del puesto que ella ocupa en la organización.

¿Por qué se generan este tipo de comportamientos por parte de la mujer para con los varones? En primera instancia, lo que puede llegar a generar este tipo de comportamientos entre jefa-colaborador se asocia con la relación histórica y del hecho que el cuerpo femenino es portador de las marcas experimentadas, ya sea como formas de opresión, exclusión, competencia o discriminación y, en la performance de algún rol, la mujer como mecanismo de defensa puede ejercer poder para no ser vulnerable. En este caso, la jefa tuvo la posibilidad de ejercer ese tipo de poder a partir del rango que tiene en la empresa. Además, se reflejó la separación entre mujeres; por ejemplo, cuando la situación es más notaria al estar presente una mujer.

Lo anterior es una forma de comprender el poder, en el entendido que cuando el sujeto mantiene relaciones con otras personas se presentarán y desarrollarán relaciones de poder que permiten comprender los contextos sociales, en este caso vinculados con el patriarcado. El contex to social que se aprecia en el ejemplo anterior tiene que ver con los cuerpos construidos por la interacción entre biología, sociedad, cultura (Lagarde, 2012) y poder. Y en estos cuerpos, se observa la diferencia anclada a un cuerpo vivido, en este caso de la mujer, que trabaja, crea, transmite y recrea la cultura, los modos de vida y las relaciones de poder en los espacios donde desempeña un rol, como lo es en el ámbito laboral. Es decir, aunque la encargada de recepción podía ejercer poder jerárquico (con base en su puesto), sin recurrir a la agresividad o hacer sentir mal al colaborador, a veces por situaciones asociadas al género, con las diferentes formas en que la mujer ha sido oprimida, la gerente de operaciones desempeñó el papel de jefa estricta para no ser vulnerable ante el personal a su cargo y demostrar que ella podía hacer las cosas sin ayuda de los demás, parecer masculina con la finalidad de justificar su ejercicio de poder.

Adicionalmente, esta diferencia no solamente es percibida por las propias mujeres sino también por sus homólogos masculinos, dando mayor peso a lo que sucede entre ellas y no a situaciones que involucran relaciones intergenéricas.

Si pones un equipo de solo mujeres no funciona como tampoco funciona un equipo de puros hombres, los conflictos van a ser diferentes, pero necesitamos unos de otros, entonces creo que el tema es más un poco de las mujeres sobre las mujeres que de los hombres sobre las mujeres (Gerente General, julio 2018).

A pesar de los problemas que se describieron, los cuales tienen que ver con los procesos de construcción sociocultural que acompañan a los hombres y mujeres, también se 
pudieron observar otro tipo de comportamientos vinculados con las relaciones de sororidad; se toman en cuenta los elementos que incorpora Riba (2016), al asociar sororidad con compañerismo y amistad.

Trabajo con puras mujeres, entonces, la verdad es que creo que sí, cuando se trata de trabajar y de esas cosas, sí es necesario el apoyo de la una a la otra, entonces no tienen problema que estemos juntas (jefa de recepción, julio 2018)

Igualmente, en la no existencia del hombre universal (Harding, 1988), existen mujeres culturalmente diferentes que "aunque tengamos diferencias entre nosotras, sabemos que tenemos nuestro apoyo” (Recepcionista, julio 2018). En este orden ideas, el sector hotelero, aunque es un espacio donde la división de género es evidente (Sarosi, 2017, Mejía et al., 2017), también puede ser una empresa turística que contribuye en la formación de alianzas y pactos entre el colectivo femenino.

Pues yo digo que como todos a veces las mujeres somos de «ay, ya nada más porque la vio, me cae mal», pero en este caso a lo mejor se llega a presentar aquí, pero pues es trabajo (Mejoramiento continuo, julio 2018), o sea, hay veces que sí nos enojamos y nos decimos de todo y, al fin y al cabo, al día siguiente estamos como si nada, todo normal, eso ya pasó y ahora a fluir todo normal (Supervisora de banquetes, julio 2018).

Esta experiencia con elementos de sororidad, puede generar nuevas pautas de relación entre conocidas y desconocidas (mujeres que laboran en empresas de hospedaje), y aunque haya áreas donde el número de mujeres sea mayor, eso no implica que sea difícil e imposible trabajar. Tal es caso de la experiencia que compartió una camarista cuando se le preguntó cómo es la relación entre ellas en el área.

Ah, pues nos tomamos nuestro café, no te voy a decir que no, nos tomamos un café, platicamos qué tenemos, porque a veces tú llegas con una carota, y ellas (compañeras de trabajo) te preguntan "qué tienes, qué te pasa, te notamos callada», "¿sabes qué?, pues me pasó esto, me pasó el otro, pues ya las metí» y ellas te dicen "cómo es posible, pues ahora cuídate». [...] Entonces, tú tratas también de apoyarlas, de darles un apoyo porque pues tampoco puedes estar esquivando, ¿no? (Camarista, julio 2018)

Son capaces de formar alianzas "porque independientemente de lo laboral llevan una muy buena relación de amistad (camaristas), por ejemplo, se pueden estar agarrando del chongo (pelearse), por así decirlo, y al poco ratito "oye, ¿vas a comer?»" (Gerente de Recursos Humanos, julio 2018). También lo observó la jefa de recepción.

Yo lo he visto con mis compañeras (camaristas) que llegan a las ocho, pero a veces a las ocho todavía no hay habitaciones vacias, entonces van por sus carritos, se toman el café, "que yo traje pan», como que tienen ese espacio, después a la hora 
de la comida igual, cuando salen igual, en el camino se van juntas, toman su camión juntas (jefe de recepción, julio 2018)

En el entendido que las relaciones intragenéricas parten de un proceso de construcción y performatividad sociocultural de los cuerpos (mujer-femenino), el turismo también tiene su papel en esta performance, al ser filtro de la cultura, el poder y el cuerpo. En primera instancia, el espacio donde se llevan a cabo las representaciones es la empresa turística (hotel), en segunda, es a partir de aspectos como la distribución del personal y la toma de decisiones que se influye en el cómo de las relaciones. Por ejemplo, cuando se cuestionó a las personas sobre a qué se debe que las relaciones entre mujeres sean algunas veces conflictivas, su respuesta la han asociado a dos aspectos: la cultura y el liderazgo. "Yo creo que sería cultural y del líder ¿no?, tenemos mucho que ver como líderes cómo enfocamos a la gente, entonces en ese aspecto sí es como la cultura que ya se trae desde casa, la ideología y posteriormente el líder" (Gerente de Recursos Humanos, julio 2018).

Adicionalmente, las personas con cargos de mayor jerarquía influyen y aportan en dichas relaciones.

Es complicado que se lleven, pero no es imposible, yo creo que el reto para los jefes departamentales está en el que conozcas a tu gente aun cuando tenemos un departamento que son la mayoría mujeres que en este caso es ama de llaves, ya sabes cómo es la personalidad de cada una de ellas [...] yo creo que sí pueden convivir, que sí pueden verse como compañeras de trabajo (Gerente de Recursos Humanos, junio 2018).

Hecha la observación anterior, una gerente de Recursos Humanos de un hotel comentó que sí hay conflictos, principalmente en el área de camaristas, pero procuran integrarlas, hacen juntas de integración, desayunos de integración, todo eso con la finalidad de que haya un mejor clima de trabajo; es decir, no se pretende separarlas o contratar varones para que haya un equilibrio y "menos hormonas femeninas", sino que se busca la manera de no anular las características comunes. Este tipo de iniciativas suceden principalmente en hoteles de 4 y 5 estrellas.

A partir del modelo de análisis, así como de los resultados de las entrevistas, observación participante y no participante y de las conversaciones informales, las relaciones intragenéricas pueden verse desde dos puntos (véase figura 3 ).

El primero se asocia cuando el poder, a través de una voluntad, como es el patriarcado, atraviesa la cultura y lo refleja en el cuerpo; es decir, se puede observar en cómo el cuerpo vivido de la mujer recrea los modos de vida y las relaciones con sus pares a partir de las construcciones socioculturales establecidas por el patriarcado, como es el hecho que entre mujeres la relación es conflictiva y se transmite como algo "normal".

En otras palabras, este punto de vista se vincula con que el cuerpo "trabaja, crea, transmite y recrea" la cultura, los modos de vida, las representaciones socioculturales. Por ejemplo: al preguntarles a las mujeres de quién han aprendido a ser mujer, su respuesta estuvo asociada, principalmente, a su madre, así como a otras mujeres que estuvieron o están en su vida y, en menor medida a su padre. 


\section{Figura 3 \\ RELACIONES INTRAGENÉRICAS A PARTIR DEL CUERPO, CULTURA Y PODER}
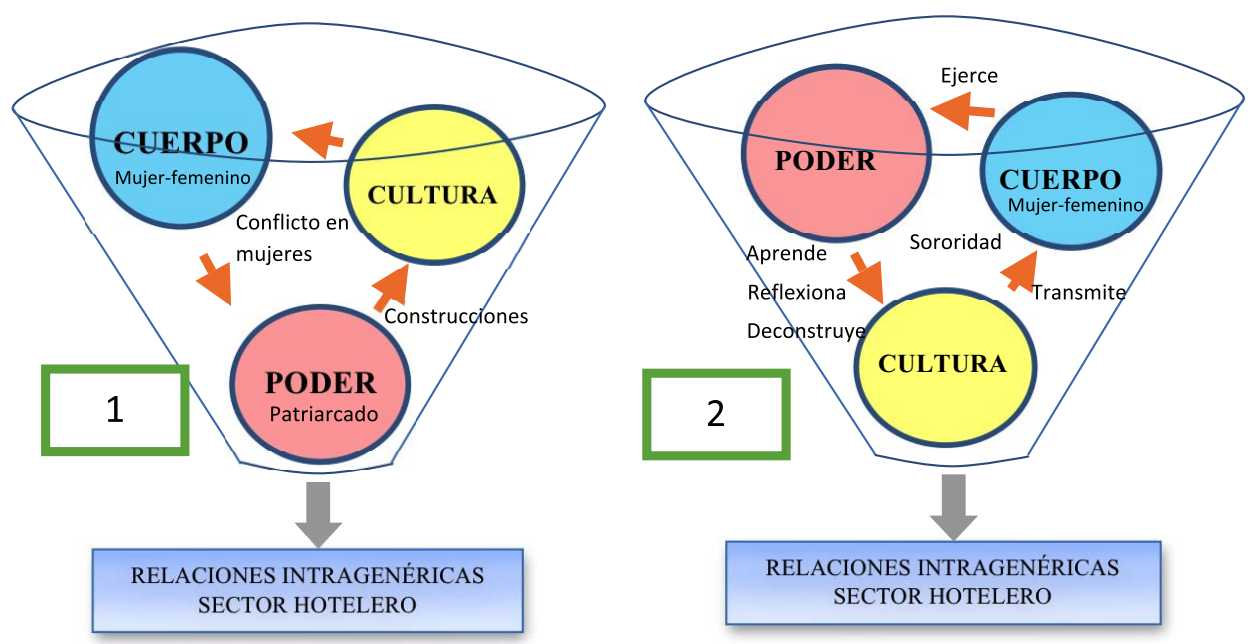

Fuente: Elaboración propia con base en Tepichín, Tinat y Gutiérrez (2010), Lamas (2017), Amigot y Pujal (2009).

Entonces, al considerar que las mujeres reproducen y son portadoras de aspectos vinculados con el patriarcado, se asocia que este tipo de relaciones entre mujeres se transmite como algo normal considerando que el cuerpo de la mujer está marcado por situaciones como la competencia. Tal es el caso de las camaristas que no estaban de acuerdo con que una de sus compañeras hubiese tomado el puesto de ama de llaves. Una forma de ver cómo ejercieron poder fue cuando el ama de llaves expresó su incomodidad con la actitud de sus colegas porque su trabajo depende de los resultados y actividades que hagan sus compañeras.

El segundo punto se refiere a cuando la cultura atraviesa el cuerpo y éste ejerce poder; es decir, se propone que el cuerpo "aprenda, reflexione, de construya y transmita" la cultura, los modos de vida, las representaciones socioculturales con la intención de transformar la visión histórica del patriarcado. Este tipo de situaciones se observó cuando la gerente de Recursos Humanos decidió implementar actividades de integración con ellas; es decir, cómo a pesar de las construcciones socioculturales, ella ejerce poder en el sentido de cambiar "lo histórico" para dejar de anular lo común.

Aunado a lo anterior, un hecho asociado con esta figura es el tema de sororidad. El cuerpo femenino es atravesado por la cultura (construcciones socioculturales, modos de vida, relaciones de poder) y una forma de ejercer su poder es cuando hay apoyo entre ellas, cuando se reconocen las propias limitaciones y las posibilidades de construir con las otras, sus iguales.

Un caso sobre este tema se suscita en España con las Kellys (las que limpian los hoteles), quienes han hecho asociaciones para informarse, estar al pendiente de su situación y condición laboral, así como para trabajar en conjunto (Alba Sud, 25 de febrero de 2018). Este tipo de acciones aporta al feminismo como voluntad de poder. Sin embargo, 
en los hoteles de este estudio no se han logrado desarrollar relaciones de sororidad, sino relaciones con elementos de sororidad como el compañerismo y la amistad. En algunas empresas se comienza a trabajar en iniciativas que contribuyen a la generación de alianzas y a reconocer lo común entre el colectivo femenino; por ejemplo, las reuniones que se llevan a cabo con ellas, las actividades de integración. Aunado a lo anterior, se presenta una situación que nace de forma natural, a través de las pláticas que ellas tienen; por ejemplo, durante un descanso en la limpieza de habitaciones, tres camaristas comentaron que después de su jornada laboral tenían que llegar a lavar, preparar comida y atender su casa. Una de ellas mostró malestar en su espalda debido a que le había tocado limpiar habitaciones con cama King Size (cuando hay este tipo de camas, se requiere mayor esfuerzo físico para poner sábanas y cobijas). Entonces, este tipo de conversaciones aportan a la generación de alianzas porque se identifican entre ellas, comparten lo común y además puede contribuir a que se encuentren socialmente con el rol que desempeñan (mujer, madre, colega, aliada).

Finalmente, en ambos puntos, el turismo, en este caso a partir de una empresa turística (hotel), tiene el papel de filtro. Ahí los hombres y mujeres llevan a cabo una performance que como se pudo observar: 1) las mujeres desempeñan papeles tradicionales, al concentrarse principalmente en puestos vinculados con lo "propio" para lo femenino y, en menor medida, en puestos de mayor jerarquía; 2) la relación entre ellas se asocia con la "enemistad histórica"; 3) aunque se parte de construcciones socioculturales que son recreadas por un cuerpo, se busca inconscientemente o no, una alianza, ese apoyo que se da por hecho que se tiene y se tendrá; 4) esto representa una oportunidad para las empresas de hacer políticas desde la perspectiva de género y 5) las mujeres se identifican entre ellas, se pueden generar relaciones de sororidad.

\section{CONCLUSIONES}

En el tema de las relaciones intragenéricas, las mujeres no solo se encuentran en disputa y es "difícil" trabajar con un equipo integrado por ellas, sino también se presentan situaciones ligadas con sororidad; es decir, hermandad entre ellas, donde lo común no es anulado sino al contrario, es vivido, creado, transmitido y recreado. Esto dependerá por una parte de la distribución del personal en cada área de trabajo. En esta investigación las actividades que realizan las mujeres en los hoteles (camaristas-limpieza, cocina-preparación de alimentos y recepción-atención a personas) están vinculadas con los roles que las mujeres desempeñan en el hogar.

Aunado a lo anterior, en los trece hoteles de este estudio, las mujeres son quienes conforman en mayor medida la plantilla laboral, así que estas empresas de hospedaje representaron un espacio óptimo para estudiar la performance que se lleva a cabo y los tipos de relaciones intragenéricas que se presentan entre el colectivo femenino. Por ejemplo, el área donde se apreciaron este tipo de relaciones fue ama de llaves (camaristas); en primera instancia porque el departamento está conformado principalmente por mujeres. Además, es reconocida por el personal de los hoteles como un área de "conflictos y rivalidad" entre ellas.

Con base en lo anterior, se observó como el cuerpo, en este caso de las mujeres, a partir de la cultura y el poder, no solo vive sino también transmite las construcciones de género y los modos de vida al reflejarse en las relaciones vinculadas con la enemistad histórica, competencia, rivalidad y disputa entre el colectivo femenino. 
Aunque en esta convivencia y concentración de mujeres en un puesto (camaristas) refleja actitudes de "disputa", también se vuelve un área de oportunidad para romper con los mandatos de una voluntad de poder como lo es el patriarcado, esto permite observar y demostrar que un equipo de mujeres tiene resultados favorables no solo operacionales (ej. tener listas las habitaciones) sino también en resultados que pueden influir en las políticas de las empresas para trabajar desde la perspectiva de género, refiriéndose principalmente, a una equidad, que aunque no es un tema que se aborda en el texto, si es una línea de investigación que se exhorta a seguir.

En las empresas de este estudio se observó que, aunque las relaciones entre mujeres se asocian a conflictos, también en algunos casos incorporan elementos de la sororidad como es el compañerismo y la amistad. Sin embargo, se requiere trabajar más para lograr alianzas entre ellas y que estos pactos nazcan desde y para beneficio de las mujeres con la finalidad de generar relaciones de sororidad. Por ejemplo, como lo que sucede en el contexto español con la formación de federaciones y asociaciones lideradas por mujeres. Algunos hoteles de este estudio, sobre todo de cuatro y cinco estrellas, están trabajando en actividades que beneficien las relaciones entre el colectivo femenino, tal es el caso de actividades de integración, iniciativas que pueden contribuir a la generación de alianzas entre mujeres.

También, es importante mencionar que en esta posibilidad de que la mujer se encuentre con otras mujeres, de mirarlas convertidas en ellas, es un camino que influye para que se reconozcan las semejanzas con otras y se disminuya la brecha de lo no común; es decir, de reconocer las propias limitaciones y posibilidades para seguir construyendo alianzas con otras mujeres, ¿será una etapa que nos lleve a una nueva forma de relaciones?

En suma, si se parte de las construcciones socioculturales o de la voluntad de poder, ¿qué contenido de género se quiere construir y tener en la hotelería? Es en las relaciones de sororidad, donde se encuentra la posibilidad de contribuir a desarticular, además de la enemistad histórica mujer-mujer, la opresión patriarcal entre los géneros masculino-femenino.

Finalmente, este estudio aporta a una línea de investigación en el turismo sobre las relaciones intragenéricas (entre mujeres), que se ha investigado en menor número en comparación con el tema de las relaciones intergenéricas (situaciones y condiciones entre mujer-varón). Asimismo, en el contexto mexicano, se exhorta a fortalecer el vínculo y trabajo entre academia y las empresas turísticas.

\section{BIBLIOGRAFÍA}

ALBA SUD (25 de febrero de 2018): Feminización del trabajo turístico y precariedad laboral [vídeo]. Disponible en: http://www.albasud.org/noticia/es/1017/feminizaci-ndel-trabajo-tur-stico-y-precariedad-laboral.

AMIGOT, P. y PUJAL, M. (2009): «Una lectura del género como dispositivo de poder», Sociológica, año 24, $\mathrm{n}^{\circ}$ 70, pp. 115-152.

ANGROSINO, M. (2012): Etnografía y observación participante en Investigación Cualitativa. Madrid, Morata.

BAÑUELOS, D., HUESCA, L. y HORBATH, J. E. (2017): «Estudio de la segregación ocupacional por razón de género en el sector turístico de México», El Periplo Sustentable, $\mathrm{n}^{\circ} 33$, pp. 159-191. 
BBC Mundo (9 de marzo de 2018): "Sororidad, la palabra que plantea una especial forma de apoyo entre las mujeres". $B B C$. Disponible en: https://www.bbc.com/mundo/noticias-43339307.

BEAUVOIR, S. [1949] (2017): El Segundo Sexo. Madrid, Cátedra.

BLANCO, M. (2006): « ¿Y las relaciones de género?», Cuadernos Inter.c.a.mbio sobre Centroamérica y el Caribe, $\mathrm{n}^{\circ}$ 4, pp. 161-169.

BOONE, J., VELLER, T., NIKOLAEVA, K., KEITH, M., KEFGEN, K. y HOURAN, J. (2013): «Rethinking a glass ceiling in the hospitality industry», Cornell Hospitality Quarterly, vol. 54 (3), pp. 230-239.

BUQUET, A., COOPER, J., MINGO, A. y MORENO, H. (2013): Intrusas en la Universidad. México, Universidad Nacional Autónoma de México.

BUTLER, J. (1998): «Actos performativos y constitución del género: un ensayo sobre fenomenología y teoría feminista», Debate Feminista, vol. 18, pp. 296-314.

CACIQUE, I. (2008): «Participación en el trabajo doméstico de hombres y mujeres en México», Papeles de Población, $\mathrm{n}^{\circ}$ 14, pp. 173-200.

CAÑADA, E. (2015): Las que limpian los hoteles. Historias ocultas de precariedad laboral. España, Icaria Editorial.

CAÑADA, E. (30 de julio de 2014): "Las que limpian los hoteles". El País. Disponible en: https://elpais.com/elpais/2014/07/30/alterconsumismo/1406706574_140670.html

CARVALHO, I., COSTA, C., LYKKE, N. y TORRES, A. (2019): «Beyond the glass ceiling: Gendering tourism management», Annals of Tourism Research, vol. 75, pp. 79-91.

COSTA, C., CARVALHO, I. y BREDA, Z. (2011): «Gender inequalities in tourism employment: the Portuguese case», Revista Turismo y Desenvolvimiento, vol. 15, pp. 39-54.

DÍAZ, I. A. (2010): «Ecoturismo comunitario y género en la Reserva de la Biosfera de los Tuxtlas» (México). Pasos. Revista de Turismo y Patrimonio Cultural, vol. 10 (5), pp. 531-542.

DÍAZ, I. A., LOZANO, M. C. y MONTIEL, J. C. (2018): «Liderazgo transformacional y género en hoteles de 4 y 5 estrellas de Tijuana (México)», Cuadernos de Turismo, $\mathrm{n}^{\circ} 42$, pp. 137-160.

DÍAZ-CARRIÓN, I. A. (2013): «Mujeres y mercado de trabajo del turismo alternativo en Veracruz», Economía, Sociedad y Territorio, vol. 13 (42), pp. 351-380.

DÍAZ-CARRIÓN, I. A. (2014): «Ecoturismo y vida cotidiana de las mujeres en Sontecomapan (Veracruz, México)», Cuadernos de Turismo, n 34, pp. 60-88.

DURÁN, J. (22 de enero de 2016): "Las Kellys salen de la habitación para denunciar su explotación laboral”. Diagonal Periódico. Disponible en: https://www.diagonalperiodico.net/global/29076-kellys-denuncian-explotacion-laboral-limpieza-habitacioneshoteles.html.

ESCUDERO, K. Y., SERRANO, R. C., SEGRADO, R., SERRANO, C. y LÓPEZ, E. (2017): «Empoderamiento de la mujer en establecimientos de hospedaje en Metepec Estado de México». Gran Tour, n ${ }^{\circ}$ 15, pp. 3-25

FERNÁNDEZ, M. y MARTÍNEZ, L. (2010): «Participación de las mujeres en las empresas turísticas privadas y comunitarias de Bahías de Huatulco, México. ¿Hacia un cambio en el rol de género?», Cuadernos de Turismo, n 26, pp. 129-151. 
FIGUEROA-DOMECQ, C., PRITCHARD, A., SEGOVIA-PÉREZ, M., MORGAN, N., y VILLACÉ-MOLINERO, T. (2015): «Tourism gender research: A critical accounting», Annals of Tourism Research, vol. 52, pp. 87-103.

FOUCAULT, M. (1991): El sujeto y el poder. Bogotá, Carpe Diem Ediciones.

FULLER, N. (2010): «Lunahuaná, un destino turístico. Transformaciones en la composición social, economía familiar y relaciones de género», Pasos. Revista de Turismo y Patrimonio Cultural, vol. 8 (2), pp. 293-304.

GIBBS, G. (2007): «Codificación temática y categorización», en El análisis de datos cualitativos en Investigación Cualitativa. Madrid, Ediciones Morata, pp. 63-82.

GOBIERNO DEL ESTADO DE MÉXICO (2018): Plan de Desarrollo (2017-2023). Disponible en: http://edomex.gob.mx/sites/edomex.gob.mx/files/files/PDEM20172023. pdf.

HARDING, S. (1988): «Is there a Feminist Method?», en Feminism y Methodology. USA, Indiana University Press, pp. 1-14.

INDINA, N. (2016): «La perspectiva de género en investigaciones sociales», en Verschuur, $C$ y Hainard, F. Des brèches dans la ville. Organisations urbaines, environnemet et transformation des rapports de genre. Ginebra, Graduate Institute Publications, pp. 37-54.

INEGI (2018): Mujeres y hombres en México (2018). Disponible en: http://cedoc.inmujeres.gob.mx/documentos_download/MHM_2018.pdf.

JAIVEN, A. L. (2016): «Feminismos», en Conceptos clave de los estudios de género, volumen 1. México, Centro de Investigaciones y Estudios de Género, Universidad Nacional Autónoma de México, pp. 139-153.

LAGARDE, M. (2006): Pacto entre mujeres sororidad. Argentina: Debates. Disponible en: http://www.asociacionag.org.ar/pdfaportes/25/09.pdf.

LAGARDE, M. (2012): «Enemistad y sororidad. Hacia una nueva cultura feminista», en El Feminismo de mi vida. Hitos, claves y topías, México, Instituto de las Mujeres del Distrito Federal, pp. 461-491.

LAMAS, M. (2017): «Género», en Conceptos clave en los estudios de género, vol. 1. México, Universidad Nacional Autónoma de México, Centro de Investigaciones y Estudios de Género, pp. 155-169.

LARA, M. A. (1993): Inventario de Masculinidad-Femineidad IMAFE. México, Editorial El Manual Moderno, S. A. de C. V.

LAS KELLYS (1 de diciembre de 2018): Hotel Explotación: Las kellys, la historia de cómo dejamos de ser invisibles y nos organizamos. Asociación las Kellys. Disponible en: https://laskellys.wordpress.com/2018/12/01/hotel-explotacionlas-kellys-la-historiade-como-dejamos-de-ser-invisibles-y-nos-organizamos/.

MARTÍN, I. (2009): Dirección y gestión de empresas del sector turístico. Madrid, Pirámide. MARTÍNEZ, D. (2014): «El poder en las relaciones de género desde la perspectiva de las mujeres», en Enfermería Actual en Costa Rica, n ${ }^{\circ}$ 27. Universidad de Costa Rica. Disponible en: https://revistas.ucr.ac.cr/index.php/enfermeria/article/view/16267.

MEJÍA, R., SERRANO-BARQUÍN, R. C, LÓPEZ-CARRÉ, E. y SERRANO-BARQUÍN, H. P. (2017): «Participación de las mujeres en empresas de hospedaje del municipio de Toluca, México. Análisis de su empoderamiento», Investigaciones Turísticas, $\mathrm{n}^{\circ}$ 14, pp. 21-44. 
MENDOZA, M. M. y GONZÁLEZ, N. G. (2013): «Mujeres empresarias en el sector turístico Huatulco, Oaxaca: la mirada masculina», Gestión Turística, n 19, pp. 107-131.

MONTERO, J. (2006): «Feminismo: un movimiento crítico», Psychosocial Intervention, vol. 15 (2), pp. 167-180.

MOONEY, S., RYAN, I. y HARRIS, C. (2017): «The Intersections of Gender with Age and Ethnicity in Hotel Careers: Still the Same Old Privileges?», Gender, Work \& Organization, vol. 24 (4), pp. 360-375.

MUNAR, A. M., BIRAN, A., BUDEANU, A., CATON, K., CHAMBERS, D., DREFGE, D., NYGAARD, L. (2015): The Gender Gap in Tourism Academy: Statistics and Indicators of Gender Equality. Disponible en: http://tourismeducationfutures.org/tefigender-in-the-tourism-academy/

NAVARRO, A. (2017): "Transformación de las relaciones de género y los "deberes" sexuales en la unión conyugal. Un caso en Michoacán», Sociedades y Desigualdades, $\mathrm{n}^{\circ} 4$, pp. 22-41.

NÚÑEZ, G. (1999): Sexo entre varones, poder y resistencia al campo sexual. México, El Colegio de Sonora - PUEG/UNAM.

OMT Y ONU MUJERES (2013): Informe mundial sobre las mujeres en el turismo 2010. Disponible en: http://cf.cdn.unwto.org/sites/all/files/pdf/informe_mundial_sobre_las_ mujeres_en_el_turismo_full_text_es.pdf.

PÉREZ, M. (2009): «Sororidad. Hermandad entre mujeres», Sororidad, n 6.

PÉREZ-SÁNCHEZ, L. y RÁBAGO-DE ÁVILA, M. (2018): «Sororidad en los procesos de envejecimiento femenino», Diversitas: Perspectivas en Psicología, vol. 14, $\mathrm{n}^{\circ} 19$, pp. 13-26.

PRITCHARD, A. y MORGAN, N. (2017): «Tourism's lost leaders: analysing gender and perfomance». Annals of Tourism Research, vol. 63, pp. 34-47.

QUINTANA, L. (17 de octubre de 2018): "La sororidad como pacto político entre mujeres que luchan". El mostrador. Disponible en: https://m.elmostrador.cl/braga/2018/10/17/ la-sororidad-como-pacto-politico-entre-mujeres-que-luchan/.

RAMALIO, U. (2011): «Supuestos y realidades de las relaciones de género en la cotidianidad. Reflexiones desde la mirada de Pierre Bourdieu», Fermentum. Revista Venezolana de Sociología y Antropología, vol. 21 (60), pp. 134-146.

RENDÓN, E. (1997): «Mecanismos sociales y relaciones de género en la decisión de emigrar», Papeles de Población, $\mathrm{n}^{\circ}$ 13, pp. 37-46.

RIBA, L. (2016): «Memoriales de mujeres: la sororidad como experiencia de empoderamiento para resistir a la violencia patriarcal», Franciscanum. Revista de las ciencias del espíritu, vol. 58, (165), pp. 225-262.

RIBEIRO, M. (2004): «Relaciones de género: equilibrio entre las responsabilidades familiares y profesionales», Papeles de Población, vol. 10 (39), pp. 219-237.

RODRÍGUEZ, E. (2011): «Las mujeres que vuelan: género y cambio cultural en Cuetzalán», Perfiles Latinoamericanos, $\mathrm{n}^{\circ} 38$, pp. 115-143.

ROJAS, M., MÉNDEZ, R. y MONTERO, L. (2013): «Satisfacción laboral y relaciones de género en la Universidad», Revista Virtual Universidad Católica del Norte, $\mathrm{n}^{\circ} 40$, pp. 204-2015. 
SÁNCHEZ, A. (1995): «Reseña de: "Cultura y relaciones de género" de Norma J. Fuller Osores», Política y Cultura, $\mathrm{n}^{\circ}$ 4, pp. 227-233.

SANTERO-SANCHEZ, R., SEGOVIA-PÉREZ, M., CASTRO-NÚÑEZ, B., FIGUEROADOMECQ, C. y TALÓN-BALLESTERO, P. (2015): «Gender differences in the hopitality industry: A job quality index», Tourism Mangement, Vol. 51, pp. 234-246.

SAROSI, D. (2017): Tourism's dirty secret: The exploitation of hotel housekeepers. OXFAM Canadá. Disponible en: https://oxfamilibrary.openrepository.com/bitstream/handle/10546/620355/rr-tourisms-dirty-secret-171017-en. pdf? sequence $=1 \&$ isAllowed $=\mathrm{y}$.

SECTUR EDO. MÉX. (2018): Directorio de hospedaje de Toluca. México, Secretaría de Turismo del Estado de México.

SEGOVIA-PÉREZ, M., FIGUEROA-DOMECQ, C., FUENTES-MORALEDA, L. y MUÑOZ-MAZÓN, A. (2019): «Incorporating a gender approach in the hospitality industry: Female executives’ perceptions», International Journal of Hospitality Management, Vol. 76, pp. 184-193

SPARRER, M. (2003): «Género y turismo rural. El ejemplo de la Costa Coruñesa», Cuadernos de Turismo, $\mathrm{n}^{\circ} 11$, pp. 181-197.

SWAIN, M. (1995): «Gender in Tourism», Annals of Tourism Research, vol. 22 (2), pp. 247-266.

TEPECHIN, A. M, TINAT, K. y GUTIÉRREZ, L. (Coords. ) (2010): Relaciones de género. Primera edición. México, El Colegio de México.

UNWTO (06 de marzo de 2019): Turismo: las mujeres toman la iniciativa. Disponible en: https://www.unwto.org/es/press-release/2019-03-06/turismo-las-mujeres-toman-lainiciativa

UNWTO (2018): "Sin equidad no hay desarrollo": Empoderamiento de la mujer en el turismo, tema central de la reunión ministerial de las Américas. Disponible en: http:// media.unwto.org/es/press-release/2018-04-16/sin-equidad-no-hay-desarrollo-empoderamiento-de-la-mujer-en-el-turismo-tema.

UNWTO (2019): Global Report on Women in Tourism. Second edition. Available at: https://www.e-unwto.org/doi/book/10.18111/9789284420384

VALEN, K. (2 de diciembre de 2007): "My Sorority Pledge? I Swore Off Sisterhood". The New York Times. Disponible en: https://www.nytimes.com/2007/12/02/fashion/02love. html.

VALEN, K. (2010): The twisted sisterhood. Unraveling the dark legacy of female friendships. New York, Ballantine Books.

VALLÉS, M. S. (2009): Entrevistas Cualitativas. Madrid, Centro de Investigaciones Sociológicas.

VIZCAÍNO-SUÁREZ, L. P, SERRANO-BARQUÍN, R., CRUZ-JIMÉNEZ, G. y PASTOR-ALFONSO, M. J. (2017): «Turismo, alfarería y trabajo femenino en el Pueblo Mágico de Metepec, México». Pasos Revista de Turismo y Patrimonio Cultural, vol. 15 (2), pp. 391-407.

WALTERS, T. (2018): «Gender equality in academic tourism, hospitality, leisure and events conferences». Journal of Policy Research in Tourism, Leisure and Events, vol. 10 (1), pp. 17-32. 